INVESTIGACIÓN

http://dx.doi.org/10.15198/seeci.2017.43.53-68

Recibido: 18/04/2017 Aceptado: 05/06/2017

\title{
FORMACIÓN DEL PROFESORADO EN CUESTIONES DE GÉNERO
}

\section{TEACHER TRAINING ON GENDER ISSUES}

Inmaculada Gómez Jarabo ${ }^{1}$ : Universidad Complutense de Madrid. España. inma.gomez@edu.ucm.es

Primitivo Sánchez Delgado: Universidad Complutense de Madrid. España. primi@ucm.es

\section{RESUMEN}

Se han conseguido muchos avances. Sin embargo, la mujer todavía está lejos de lograr una igualdad real con el hombre y, en algunos casos, sigue siendo objeto de agresiones físicas, psicológicas, verbales, sexuales... que, en ocasiones, tienen su origen en las ideas que histórica y culturalmente han sido transmitidas de generación en generación. En algunos sectores sociales es habitual la creencia de que la violencia de género no afecta a la infancia y la juventud, pero las últimas investigaciones muestran una situación diferente: los centros escolares son el escenario de los primeros abusos de género. La labor del profesorado es esencial en la detección y tratamiento de la violencia, la mejora de la convivencia escolar y la formación y sensibilización en cuestiones de género. La investigación que se presenta en este artículo pretende desvelar si el profesorado está preparado para ello y si la formación del profesorado tiene en cuenta esos aspectos. Aquí se muestran algunos resultados relevantes de una investigación sobre la "formación del profesorado para el tratamiento educativo de los conflictos sobre diversidad cultural y de género", en la que se utilizó una metodología mixta de investigación. Los resultados ponen de manifiesto que existen carencias importantes en la formación del profesorado en relación a cuestiones de género, convivencia entre sexos, gestión de conflictos y violencia.

PALABRAS CLAVE: Género - convivencia - conflicto - violencia - diversidadeducación del profesorado - desarrollo profesional de docentes.

\footnotetext{
${ }^{1}$ Inmaculada Gómez Jarabo: Licenciada y Doctora en Pedagogía por la Universidad Complutense de Madrid. Profesor asociada al Departamento de Didáctica y Organización Escolar de la UCM

Correo: inma.gomez@edu.ucm.es
} 


\section{ABSTRACT:}

There have been many advances. However, women are still far from achieving real equality with men and, in some cases, they still undergo physical, psychological, verbal, sexual assaults... aggressions that, sometimes, have their origin in the ideas historically and culturally transmitted from generation to generation. Some people believe that domestic violence does not affect the new generations, but the latest research shows us a very different situation: schools are the first stage of gender abuse. Therefore, teachers' work is essential in detecting and treating violence, in improving school life and in training and raising awareness on gender issues. The piece of research contained herein intends to reveal whether teachers are ready for that and whether teacher training takes these issues into account. In this article, we show some of the most relevant results of research on "teacher training for the educational treatment of conflicts on cultural diversity and gender". The results state, in consistence with others previous pieces of research, the fact that there are significant gaps in the training of teachers in gender, gender coexistence, conflict management and violence issues.

KEY WORDS: Gender - coexistence - conflict - violence - diversity - teacher education - teachers' professional development.

\section{Cómo citar el artículo}

Gómez Jarabo, I. y Sánchez Delgado, P. Formación del profesorado en cuestiones de género. [Teacher training on gender issues]. Revista de Comunicacion de la SEECI, 43, 53-68. doi: http://dx.doi.org/10.15198/seeci.2017.43.53-68 Recuperado de http://www.seeci.net/revista/index.php/seeci/article/view/474

\section{INTRODUCCIÓN}

Hemos vivido en un sistema patriarcal, en el que a las mujeres se les asignaban funciones de cuidado de la familia y el hogar dentro del espacio doméstico y a los hombres las de trabajar fuera del hogar para mantener a la familia. La literatura ofrece afirmaciones que dan una idea de lo que se ha considerado ser mujer durante años:

Las parientas de mi padrastro decían, por tanto, que yo no era buena para nada porque no sabía planchar, ni cocinar, ni calcetar; porque no lavaba los cristales, ni hacía las camas, ni barría mi cuarto. Según ellas, yo necesitaba veinte criadas y me daba el tono de una princesa. Ridiculizaban también mi afición al estudio y me llamaban la Doctora... (Gómez de Avellaneda, citado por Catena, 1989, p.169).

Ese reparto de funciones, hacía que las mujeres recibieran una educación diferenciada en espacios, contenidos e incluso en cuanto a los niveles educativos a los que podían acceder. Ha habido un gran cambio social respecto al papel del hombre y la mujer. Hoy las mujeres pueden acceder a todos los niveles educativos y 
no existe un currículum diferenciado en función del sexo. Afortunadamente, ideas como la siguiente han quedado atrás para una gran parte de la población: "Las mujeres nunca descubren nada; les falta el talento creador reservado por Dios para inteligencias varoniles" (Pilar Primo de Rivera, 1942, citada por Otero, 2001, p.176). No obstante, las mujeres siguen accediendo en menor medida a los estudios más prestigiosos y encuentran más trabas a la hora de encontrar un trabajo. Muchas niñas y niños siguen jugando a juegos o con juguetes que tradicionalmente se asignaban a su sexo. En palabras de Subirats (1994): La igualdad formal no va acompañada de igualdad real, pues a pesar de que las mujeres pueden acceder a los mismos estudios y trabajos, todavía no lo hacen.

El acceso universal de la mujer a la educación, junto con los grandes cambios que se han producido en los últimos años respecto a su posición social y respecto a los estereotipos de género, podrían hacer pensar que la violencia de género ha sido erradicada, pero nada más lejos de la realidad. Continuamente salen a la luz noticias de mujeres insultadas, violadas, agredidas física y psicológicamente.

Muchas investigaciones muestran que la violencia de género no es exclusiva de personas de edad avanzada, que posiblemente se criaron con otros ideales y valores, sino que afecta a niños, niñas y jóvenes. La mayor igualdad entre personas de diferente sexo no conlleva un total respeto entre ellas ni una convivencia exenta de problemas.

Así, Pérez y Montalvo (2011, p. 90-92) alertan de lo siguiente:

En el año 2009, de las 55 víctimas por violencia de género cinco tenían entre 18 y 20 años y once entre 21 y 30 años. Franjas de edad en las que cada vez es más frecuente que se produzcan muertes por violencia machista y en las que, sobre todo, los diferentes estudios especifican que lo común es encontrar constantes episodios de violencia psicológica entre algunos grupos de adolescentes y jóvenes. (...) En general, la población joven considera que el maltrato sólo tiene relación con agresiones físicas graves, obviando otras formas de violencia de género. La mayoría de los chicos y chicas piensan que el problema se da en mujeres casadas y mayores y no en las jóvenes asociando igualmente la creencia de que los varones presentan dificultades para controlar la agresividad por ser ésta un tema hormonal (...) La creencia entre la juventud de que la violencia de género sólo se da en personas mayores debe preocuparnos. Los y las jóvenes no imaginan que la bofetada 0 los insultos que se producen en su momento presente se corresponden con el mismo fenómeno que sufre la señora que ha muerto por una paliza y que sale en las noticias de televisión.

En la misma línea, Díaz Aguado y Carvajal (2011) ponen de manifiesto datos que, como poco, son inquietantes. Sirvan de ejemplo los siguientes:

1. Un $24,7 \%$ de los y las adolescentes participantes en la investigación se muestra algo de acuerdo con que "el hombre que parece agresivo es más atractivo" y un $7,1 \%$ y un $2 \%$, bastante y muy de acuerdo. 
Gómez Jarabo, I. y Sánchez Delgado, P. Formación del profesorado en cuestiones de género

2. Un $30,7 \%, 6,5 \%$ y un $2,8 \%$ de los y las adolescentes participantes en la investigación están algo de acuerdo, bastante de acuerdo y muy de acuerdo respectivamente con que "es correcto pegar a alguien que te ha ofendido".

3. Un $29,7 \%$ de los y las adolescentes participantes en la investigación se encuentran algo de acuerdo, bastante de acuerdo y muy de acuerdo con que "para tener una buena relación de pareja es deseable que la mujer evite llevar la contraria al hombre".

4. Al preguntar a los y las adolescentes si sus parejas "han intentado aislarles de sus amistades" un $15,1 \%$, un $3,3 \%$ y un $2,8 \%$ respectivamente responden que "a veces", "a menudo" $y$ "muchas veces".

5. Un $2,7 \%$ de los y las adolescentes participantes en la investigación afirmaron que sus parejas les habían pegado "a veces", seguido de un $0,4 \%$ que respondieron que "a menudo" y otro $0,4 \%$ que respondió "muchas veces".

La última investigación del Centro Reina Sofía sobre Adolescencia y Juventud (2015) muestra que el $33 \%$ de chicos adolescentes y jóvenes españoles (12-24 años) están muy, bastante y algo de acuerdo con: "Está bien que los chicos salgan con muchas chicas, pero no al revés".

Son ejemplos de que se está muy cerca de justificar la violencia o de valorar la visión androcéntrica, basada en la mentalidad machista. Se puede pensar que son porcentajes bajos, pero un mínimo porcentaje de respuestas en ese sentido ya debe inquietar, pues quiere decir que hay un número de adolescentes en situación de riesgo y que, por tanto, los malos tratos en la pareja no se restringen a la vida adulta. El cambio generacional detectado en los y las adolescentes no es suficiente para erradicar la violencia de género.

Diversas investigaciones muestran que la violencia de género no es un problema aislado, sino que afecta a todos los países, a todos los ámbitos de la sociedad y a todas las franjas de edad. Todas las personas deben estar informadas y sensibilizadas para trabajar en su erradicación. $Y$ ahí es donde la escuela juega un papel fundamental. Durante la infancia y la adolescencia se asientan las bases de las relaciones de pareja, lo que hace que sea fundamental intervenir lo más tempranamente posible en materia de género.

Es imprescindible incluir en el currículum escolar contenidos y acciones específicas para trabajar los estereotipos, el autocontrol, la autoestima, las habilidades sociales y la construcción de una identidad propia y positiva... La violencia de género únicamente se puede erradicar con prevención y sensibilización. Como indica el Seminario Galego Educación Paz (2006, p. 125):

La educación colabora formando mentalidades, identidades personales y sociales, formando personas más sensibles capaces de situarse de otra manera delante de los problemas, convirtiéndolos en un elemento básico para la igualdad, el perfeccionamiento y la optimización de los seres humanos. 
Se requiere una educación que, asumiendo las diferencias entre chicos y chicas, y reconociendo la diversidad, permita que tanto unos como otras puedan desarrollarse con los mismos derechos y obligaciones. Esto pasa por cuestionar el modelo de masculinidad que se ha venido transmitiendo de generación en generación y que consistía en asociar la agresividad, la fuerza y la violencia a la identidad masculina. Según de Celis (2011, p. 73), la socialización masculina pasaba "por demostrar la fuerza y la agresividad, y por adquirir el derecho a dominar y controlar a través de ella". Hay que huir de ese modelo, condenando todas las formas de violencia, y mostrando a niños, niñas y adolescentes que ser varón no tiene por qué estar asociado a la fuerza o al ejercicio de la violencia, sino que puede estar asociado a la empatía, el diálogo, la igualdad, el cariño, la sensibilidad...

Para prevenir el sexismo y la violencia de género en las escuelas, el primer paso es que el profesorado sea consciente de sus propias vivencias, pensamientos y actuaciones y esté concienciado de la necesidad de atender a la diversidad y de trabajar para lograr una igualdad de género efectiva. ¿Está el profesorado sensibilizado? ¿Se está formando al profesorado para fomentar una convivencia pacífica entre chicos y chicas? ¿Y para intervenir en los casos en los que esa convivencia pacífica se ve amenazada?

Para contestar a esas y otras preguntas, se desarrolló una investigación sobre la formación del profesorado para el tratamiento educativo de los conflictos sobre diversidad cultural y de género. A continuación analizamos los resultados más relevantes referidos al género.

\section{OBJETIVOS}

Tras analizar diferentes noticias aparecidas en los medios de comunicación social, entablar conversaciones con colegas de profesión, alumnos y alumnas de diferentes titulaciones de educación y realizar una amplia consulta bibliográfica, parecía que existían importantes carencias en la formación de las titulaciones de Psicopedagogía, Pedagogía, Educación Social, Educación Infantil y Educación Primaria respecto a género, convivencia, conflictos, violencia...

Se pretendía conocer la percepción del profesorado (en formación y en activo en Educación Infantil, Primaria, Departamentos de Orientación y Equipos de Orientación Educativa y Psicopedagógica) sobre si existían carencias en su formación inicial y permanente, y sobre la influencia que éstas pueden tener en su desarrollo profesional.

El objetivo sobre la perspectiva de género es: "Conocer las actitudes y el grado de preparación que tienen los profesionales de la educación para atender a la diversidad de género, promover una convivencia saludable e intervenir en situaciones de conflicto y hacer propuestas de mejora". 
Gómez Jarabo, I. y Sánchez Delgado, P. Formación del profesorado en cuestiones de género

\section{METODOLOGÍA}

Se pretende profundizar en lo que pasa en las aulas de formación del profesorado y en los centros educativos. Se trata de conocer si los participantes en la investigación habían recibido o estaban recibiendo formación para lograr una convivencia pacífica, si se consideraban preparados para intervenir en situaciones violentas, qué opinión tenían acerca de su formación, qué puntos fuertes y débiles encontraban en la misma, por qué accedían o no a formación en determinados temas...

Se utilizó una metodología mixta, buscando la complementariedad y compatibilidad de lo cuantitativo y lo cualitativo, pues según Reichardt y Cook (1986), existen al menos tres razones que justifican la combinación de métodos cuantitativos y cualitativos:

1. La investigación tiene propósitos múltiples que han de ser atendidos con variedad de métodos.

2. Empleados en conjunto y con el mismo propósito puede lograrse lo que ninguno de los dos por separado lograría.

3. Ningún método está exento de prejuicios. Hay que triangular para reducir los sesgos de cada uno y someterlos a comprobación.

\subsection{Instrumentos}

En un primer momento, se utilizó un cuestionario como acercamiento a la realidad. Con el fin de que las personas participantes se expresaran con libertad sobre los aspectos que nos preocupaban de antemano y otros emergentes, se optó por un cuestionario con preguntas politómicas o categorizadas y abiertas.

Posteriormente, se realizaron grupos de discusión y entrevistas, con el objetivo de reducir los sesgos, profundizar y enriquecer la comprensión del ámbito de estudio. Las entrevistas se hicieron semiestructuradas y en los grupos de discusión, a pesar de contar con un guión, se procuró fomentar un debate libre.

También se revisaron los planes de estudio vigentes para determinar las asignaturas en las que se abordaban los temas de la investigación.

Los instrumentos se sometieron a validación por parte de expertos en formación del profesorado, género, interculturalidad, violencia y metodología de investigación. Todas las sugerencias ofrecidas por los expertos y las expertas fueron tenidas en cuenta para la mejora de los instrumentos. Además, con el fin de comprobar que las preguntas incluidas en los instrumentos se entendían correctamente, se aplicaron previamente éstos a un pequeño grupo piloto de características análogas a lo que después sería la muestra definitiva del estudio. Todas las sugerencias realizadas, tanto por los expertos en la materia, como por las personas que colaboraron voluntariamente en la revisión de los instrumentos, fueron tenidas en cuenta para conformar el cuestionario y los guiones definitivos de entrevistas y grupos de discusión que se emplearon en la investigación. 
Gómez Jarabo, I. y Sánchez Delgado, P. Formación del profesorado en cuestiones de género.

\subsection{Muestra}

En el cuestionario participaron 204 personas de la Comunidad de Madrid (España), 40 hombres $(19,6 \%)$ y 164 mujeres $(80,4 \%)$. De las personas participantes, 66 $(32,4 \%)$ eran profesores y profesoras en activo y $138(67,6 \%)$ estudiantes de último curso de diferentes titulaciones de educación. Los estudiantes se distribuyen así: $47,5 \%$ de $4^{\circ}$ curso de Grado, $51,2 \%$ de $5^{\circ}$ curso de Licenciatura (Pedagogía y Psicopedagogía) y $1,2 \%$ de Máster.

El profesorado provenía mayoritariamente de centros públicos $(46,9 \%)$ y concertados (45,3\%). También contestó un 7,8\% de centros privados.

En las entrevistas participaron 15 estudiantes y 15 profesores: 10 estudiantes de Grados de Educación Social, Pedagogía y Magisterio y 5 de Diplomatura en Magisterio, Licenciatura en Pedagogía y en Psicopedagogía. 10 eran mujeres y 5 hombres. En cuanto al profesorado, 9 eran mujeres y 6 hombres, 3 trabajaban en Educación Infantil, 6 en Educación Primaria, 4 en Educación Secundaria y 2 en Educación Especial.

En los grupos de discusión participaron 8 estudiantes ( 4 de Grado y 4 de planes a extinguir, 6 mujeres y 2 hombres) y 9 profesionales en activo (5 mujeres y 4 hombres).

\section{DISCUSIÓN}

\subsection{Actitudes ante la diversidad.}

La información obtenida en el cuestionario, las entrevistas y los grupos de discusión permite afirmar que la mayoría de las personas participantes se muestran muy a favor de la escuela que acoge a todas las personas, sea cual sea su sexo, origen, condición social... Consideran la diversidad como algo positivo que permite el crecimiento personal y social. En algunos casos incluso se llega a opinar que "no hay otra opción, si lo que queremos es educar a ciudadanos y ciudadanas. La escuela tiene que estar totalmente normalizada y dar cabida a todas las personas que forman la sociedad" (Alumna de $5^{\circ}$ de Pedagogía) y que "eso es lo que debería ser la escuela, no deberíamos hablar de inclusiva, sino de escuela" (Alumna de $4^{\circ}$ de Educación Social).

\subsection{Formación inicial del profesorado.}

Todas las pruebas muestran que la formación para la convivencia entre sexos ha estado ausente en la preparación de la mayoría de participantes (únicamente un $27,1 \%$ afirma haber recibido algo de formación en ese sentido). Gran parte de los comentarios realizados en los cuestionarios hacen pensar que el tratamiento de ese tema depende mucho de la iniciativa o el interés personal de cada profesor o 
Gómez Jarabo, I. y Sánchez Delgado, P. Formación del profesorado en cuestiones de género profesora:

1. "A lo largo de la carrera tan solo me ha impartido clase un profesor que se ha mostrado comprometido con la causa de la igualdad de género y ha tintado sus clases de estos asuntos de manera trasversal" (Alumna de $5^{\circ}$ de la Licenciatura de Pedagogía).

2. "Hemos notado que el trabajo en estos temas se ha realizado sobre todo por el interés de los docentes" (Alumno de $4^{\circ}$ del Grado de Educación Social).

3. "Lo cierto es que sobre esta temática no se muestra nada a menos que haya algún profesor o alguna profesora a quien le interese sacarla. La formación sobre esto es muy pobre" (Alumno de $5^{\circ}$ de la Licenciatura de Pedagogía).

El anterior resultado es coincidente con el que obtuvieron el Instituto de la Mujer y la Red2 Consultores (2004), citados por Anguita y Torrego (2009, p. 20):

Los docentes en ejercicio no han recibido formación específica en materia de coeducación, y ello les lleva a pensar que no es necesario actuar en los centros educativos porque consideran que la igualdad de oportunidades entre hombres y mujeres es un hecho superado.

Romero y Abril (2008, p. 5) también obtuvieron resultados semejantes en relación a los estudios de Educación Infantil (ciclos formativos y estudios universitarios):

Algunas universidades empiezan a elaborar planes de igualdad y crean observatorios de igualdad; pero aún no es un hecho generalizado. En las facultades y centros de secundaria donde se imparten los estudios de Educación Infantil, el género no suele ser una prioridad del equipo docente. Cuando se tratan temas de género en los centros, suele ser por iniciativa individual, interés o sensibilidad de un determinado profesor.

Por otro lado, en el análisis que se hizo sobre los planes de estudio de la especialidad de maestro en Educación Infantil de las universidades españolas, se comprobó que la mayoría no disponía de asignaturas específicas sobre género y coeducación. Así, en la mayoría de los casos, en la formación inicial, cuando hay contenidos sobre género, estos se incluyen dentro de asignaturas no específicas.

Además, esta investigación ha puesto de manifiesto que el problema también parece encontrarse en que cuando se recibe formación para la convivencia entre sexos no se hace de una forma amplia:

1. "Nuestra profesora nos habló del tema, pero no entramos en profundidad ni nos dio una formación sólida respecto a ello". (Alumna de 40 del Grado de Educación Primaria)

2. "Una formación que a pesar de ser útil fue un poco escasa al tratarse de una exposición de un trabajo en grupo dentro de la asignatura" (Alumno de $5^{0}$ de la Licenciatura de Psicopedagogía).

3. "Ha sido inexistente, limitada a comentarios prototípicos en el desarrollo de algunas asignaturas". (Alumna de 40 del Grado de Educación Infantil). 
Gómez Jarabo, I. y Sánchez Delgado, P. Formación del profesorado en cuestiones de género.

4. "Solo se trata en momentos puntuales en que surge en algún debate 0 situación concreta, o comentado por encima por el profesor, pero no sirve para producir un verdadero cambio de actitud o eliminar prejuicios en la práctica ni se suele tratar más allá que de forma teórica" (Alumna de $4^{\circ} \mathrm{del}$ Grado de Educación Primaria).

Sin duda, una de las causas de no profundización en ese tema es que no aparece expresamente recogido en el plan de estudios o en la guía docente.

Al preguntar por la calidad y utilidad de la formación recibida, aunque la gran mayoría $(66,1 \%)$ consideró la formación recibida "bastante útil", un porcentaje bastante elevado (50,3\%) la calificó como "mala".

Sin embargo, las actitudes de las personas participantes hacia este tipo de formación son muy positivas (sobre todo de las mujeres). Veamos algunos comentarios al respecto:

1. "Esto debería ser algo que se trabaje desde preescolar y muy a fondo. Así luego de mayores no existirían tantos problemas en la sociedad relativos a la desigualdad entre hombres y mujeres" (Alumna de 40 del Grado de Educación Social)

2. "Sería muy útil que nos formaran para aprender a solucionar problemas relacionados con género, hoy en día el machismo está a la orden del día, las mujeres siguen cobrando menos que los hombres y la solución está en la escuela" (Alumna de $5^{\circ}$ de la Licenciatura de Pedagogía).

A la luz de los resultados obtenidos, parece que la gestión de conflictos, el tratamiento de la violencia y el conocimiento de uno mismo tampoco tienen demasiada presencia en la formación del profesorado, a pesar de la importancia que tienen de cara a evitar que nuestros propios prejuicios influyan en la forma en la que nos relacionamos con otras personas y a reconducir situaciones que sin una buena intervención podrían degenerar en negativas.

Aunque existen carencias importantes en todas las titulaciones de educación en relación a temas como el género, los conflictos, la convivencia o la violencia, parece que la titulación en la que se recibe mayor formación en esos temas es en el Grado de Educación Social. A pesar de que se recibe mayor formación, las críticas realizadas son las mismas que en el resto de casos: se considera que la formación es útil, pero insuficiente y demasiado teórica, no ofreciendo respuesta a las necesidades reales que se presentan en los centros educativos.

\subsection{Desarrollo profesional del profesorado.}

Los diferentes instrumentos de investigación muestran que la mayoría de participantes en la investigación no han accedido a formación permanente relacionada con la mejora de la convivencia entre sexos. Únicamente un $11.5 \%$ ha recibido formación permanente en relación a este tema. 
Los principales motivos por los que no se ha recibido formación son "no haber recibido oferta" $(65,1 \%)$, "no tener tiempo" $(18,5 \%)$ o el "coste económico de las actividades formativas" (4,7\%), aunque también nos encontramos con personas que aluden otros motivos como problemas de accesibilidad al lugar de impartición o no considerar necesaria esa formación.

Quienes han participado en actividades, consideran que han sido bastante útiles, aunque señalan como principales fallos: poca duración, falta de aplicación práctica (formación muy teórica y basada en casos irreales) y poca especificidad o poca adaptación a cada caso. En algunos casos, también se plantea que se trata de actividades no actualizadas o que han bajado su calidad y su oferta en los últimos años:

No existe una gran oferta de formación permanente para los profes. Cada vez existe menos oferta y siempre es sota, caballo y rey, siempre son recursos didácticos para el aula, informática en el aula y ahora con el bilingüismo, pues cursos de inglés (Profesora de Educación Especial).

La formación que las personas participantes consideran más efectiva para mejorar la convivencia o tratar educativamente los conflictos de género es la que incluye dinámicas, casos prácticos, trabajo en equipo y reflexión compartida.

\subsection{Capacitación para mejorar la convivencia y gestionar la diversidad.}

Aunque no siempre se ha recibido formación para la mejora de la convivencia o para la gestión de conflictos y prevención de la violencia, los participantes mayoritariamente $(56,7 \%)$ se sienten capacitados para mejorar la convivencia y gestionar la diversidad. Sin embargo, si diferenciamos entre las respuestas ofrecidas por el profesorado y por el alumnado, observamos bastantes diferencias: vemos como el $80,4 \%$ del profesorado reconoce sentirse muy o bastante preparado, mientras que únicamente el $46,4 \%$ del alumnado afirma estarlo.

En muchos casos se reconoce que se interviene (y se intervendría) más por sensibilidad personal o sentido común que por conocimiento científico o por haber recibido formación:

1. "Al no haber recibido formación, ¿cómo puedo saber cómo intervenir? Únicamente puedo basarme en mi sentido común" (Alumna de $4^{\circ}$ del Grado de Educación Infantil).

2. "No se me ha formado para ello, pero creo que sería más sencillo que en el caso de las culturas, pues en todos los espacios hay chicos y chicas, y ya prácticamente todos asumimos esa necesidad" (Alumno de $4^{\circ}$ del Grado de Educación Infantil).

3. "Muchas veces funcionas por sentido común, cómo lo haría yo, cómo lo voy a hacer que creo que puede funcionar y me voy a equivocar $y$ a lo mejor no funciona y lo voy a intentar de otra manera, $Y$ muchas veces a raíz de ese pruebo y me equivoco, te sale" (Profesora de PCPI). 
Gómez Jarabo, I. y Sánchez Delgado, P. Formación del profesorado en cuestiones de género.

Resulta llamativo que estudiantes y profesionales coincidan en afirmar que la preparación del profesorado para mejorar la convivencia y gestionar la diversidad depende mucho de cada persona, de su edad, de sus experiencias personales y de la formación que hayan recibido. Tiende a confiarse más en profesionales de Educación Infantil o Primaria que en los de Secundaria.

\subsection{Formación necesaria para atender a la diversidad.}

Se pretendía conocer no sólo si se había recibido formación y si ésta había sido útil, sino también cuál era la formación que se consideraba más necesaria y apropiada para atender adecuadamente la diversidad. Las respuestas proporcionadas por estudiantes y profesorado son muy coincidentes. La propuesta más repetida consiste en que la formación, si bien necesita suficiente base teórica, debe estar mucho más enfocada a la práctica (no hay que olvidar que mayoritariamente han recibido una formación teórica y con escasa o nula aplicación práctica). Se considera que habría que cambiar la concepción de muchas asignaturas para que respondieran a las necesidades de un profesional de la educación, así como que es preciso invertir en temáticas "más humanas", como las habilidades sociales, el autoconocimiento, la autoestima, el trabajo en equipo, el acompañamiento a lo largo de toda la vida, las técnicas de gestión de conflictos como la mediación... además, de en los temas de esta investigación: género, conflictos, violencia, convivencia...

Destacamos las palabras de un orientador de educación secundaria y profesor de las titulaciones de educación en la universidad:

Yo soy también profe de la universidad, pero te he de decir que estamos todavía a años luz de enseñar competencias reales de resolución de conflictos y procedimientos que estén avalados, respaldados como útiles tanto de forma clínica como por la ciencia y, en ese caso, yo creo que sí... siempre falta formación (...) Además, hay un trabajo muy importante, que es el personal, el autoconocimiento, el manejo de las propias emociones, tu propia autorregulación, tus propias habilidades sociales... ese trabajo no se hace (Orientador de Educación Secundaria).

\subsection{Situación de las aulas y actuación en las mismas.}

El profesorado alerta de que los conflictos por razón de sexo son frecuentes en los centros. Se observan gran cantidad de comportamientos machistas, tanto en alumnos y alumnas, como en familias y en profesorado:

Sí se dan esas situaciones de conflicto machista, trasnochado completamente, pero no solamente en el chico. Lo otro que yo me encuentro es que vuelven determinadas situaciones, determinadas actitudes que se suponían ya archivadísimas y cerradísimas y otra vez estamos teniendo unos problemas, unas ideologías y unas formas de actuar que son tremendas (Orientadora de Educación Secundaria).

Un profesor de PCPI resalta: "Nuestros alumnos tienden a relacionarse de forma muy 
Gómez Jarabo, I. y Sánchez Delgado, P. Formación del profesorado en cuestiones de género

convencional y muy marcadas por los estereotipos, tanto ellos como ellas, y eso puede dar lugar a problemas".

Se piensa que falta preparación para intervenir ante determinadas situaciones. Cuando esto ocurre y la persona no es capaz de solucionar un problema por sus propios medios, tiende a buscarse apoyo en el Departamento de Orientación, en otros profesionales y en la Dirección del Centro.

Son pocos los profesionales que desarrollan proyectos concretos para la mejora de la convivencia, fundamentalmente, por falta de tiempo y de motivación. Para evitar situaciones de rechazo y exclusión, así como fomentar la convivencia en las aulas, el profesorado tiende a incorporar la igualdad en su aula (utilizando lenguaje inclusivo, usando materiales adecuados, tratando determinados temas...) y a fomentar los agrupamientos heterogéneos en las tareas de clase.

\subsection{Otros aspectos destacables.}

Parte del alumnado y profesorado han señalado que, en ocasiones, se transmiten determinados mensajes no apropiados por parte del profesorado o cómo el tratamiento de género o de los conflictos en las aulas no es el más indicado. Al respecto, encontramos afirmaciones como las siguientes:

1. "En ocasiones no me ha gustado un pelo el tratamiento de género por parte de los profesores" (Alumna $3^{\circ}$ de Magisterio de Educación Física).

2. "Incluso la formación recibida, tanto en forma como en fondo, se podría catalogar de sexista y discriminatoria" (Maestra de Lengua Extranjera y Pedagoga).

Eso es preocupante porque el profesorado tiene un amplio efecto multiplicador, además de llegar a muchos estudiantes y familias a lo largo de su vida activa, muchas personas no cuestionan lo que dicen.

\section{CONCLUSIONES}

A pesar de los avances producidos en los últimos años en relación a la situación de las mujeres y a su participación en todas las esferas sociales, todavía en pleno siglo XXI seguimos viendo situaciones en las que mujeres de todas las edades son el blanco de diferentes formas de violencia. Aunque pudiera parecer que esos hechos únicamente están presentes en adultos o en personas de edad más avanzada, las últimas encuestas e investigaciones muestran una realidad muy diferente: las escuelas son testigos (a veces, mudos) de discriminaciones, insultos, vejaciones... a chicas de todo tipo de condiciones sociales, económicas y culturales. Ante esa situación, se hace preciso un trabajo profundo que nos permita prevenir las situaciones violentas en los centros educativos o intervenir educativamente cuando la prevención no ha funcionado. No en vano, la educación es "un factor esencial en la consolidación de una cultura de paz en la sociedad" (Aguilar y Castañón, 2014, p.92). 
Coincidimos con Díaz-Aguado (2002) en que, para construir la igualdad y prevenir la violencia contra la mujer, es preciso incluir la lucha contra el sexismo y contra la violencia hacia las mujeres en el currículum escolar, pues no basta con que la escuela se limite a no ser sexista. Esa inclusión en el currículum escolar debe permitir:

1. Favorecer cambios cognitivos, afectivos y conductuales, que ayuden a superar los distintos componentes del sexismo y la violencia.

2. Incrementar esfuerzos para superar la invisibilidad de las mujeres.

3. Desarrollar habilidades para identificar y rechazar los estereotipos sexistas, que contribuyen a la violencia.

Abordando la perspectiva de género en los centros escolares, estaremos dando un paso muy importante para la superación de los estereotipos sexistas que llevan a niños, adolescentes, jóvenes y adultos a justificar, emplear o silenciar la violencia hacia la mujer.

Además, como indican Gairín, Armengol y Silva (2013), existen estrechas relaciones entre el currículum escolar, los métodos de enseñanza, los sistemas de evaluación y las normas de convivencia, que influyen en la probabilidad de que sucedan comportamientos antisociales en los espacios educativos. Por ejemplo, pueden relacionarse el tiempo que el profesor dedica en el aula a procesos de grupo, a relaciones interpersonales y a cuestiones de normas, orden y disciplina con la disminución de los comportamientos disruptivos y de maltrato entre iguales. Pero para que el currículum escolar tenga en cuenta esos aspectos, previamente es preciso formar al profesorado.

Sin embargo, la formación del profesorado no siempre presta la atención necesaria a aspectos como las relaciones interpersonales o las normas de aula y de centro. Esta investigación ha mostrado que, a pesar de que el futuro profesorado y los docentes están sensibilizados con estas cuestiones y otras relacionadas, no han sido formados para ello. Esto puede limitar su capacidad de acción y hacer que no consideren necesaria la prevención o intervención en ese sentido.

Es necesario invertir esfuerzos en reforzar la formación inicial, pues falta formación sobre cuestiones de género para atender a la diversidad, para intervenir ante la violencia, para aprovechar positivamente los conflictos, para desarrollar programas de prevención de la violencia o para trabajar cooperativamente. Existen pocas asignaturas que aborden esos aspectos y, las que hay, son optativas. Por ello, es preciso invertir esfuerzos para que las relaciones entre iguales, la igualdad de oportunidades entre chicos y chicas, el trabajo en equipo y la gestión educativa de conflictos ocupen el lugar que merecen en la formación de todo el profesorado sin excepción, pues de nada sirve que se formen únicamente algunos futuros profesores.

Hay profesores que abogan por tratar estos aspectos de forma transversal. No se puede ni se debe dejar relegados temas tan importantes a la optatividad ni a la transversalidad. Eso no garantiza que todas las personas que deban recibir formación 
Gómez Jarabo, I. y Sánchez Delgado, P. Formación del profesorado en cuestiones de género

la reciban. Es fundamental introducir asignaturas específicas en la formación inicial de todos los profesionales de la educación.

Es preciso también que la formación permanente del profesorado se haga eco de esta necesidad y se incrementen las actividades en las que se aborde la perspectiva de género y, por tanto, también los conflictos, la convivencia, el trabajo en equipo... En cualquier caso, no es suficiente incluir esos temas en la formación inicial y permanente del profesorado, también debe cuidarse la forma en la que se incluyen:

1. Incidiendo en las necesidades reales de los centros educativos.

2. No limitando la formación a los contenidos conceptuales y apostando por una formación más práctica, menos memorística

3. Prestando especial atención a la dimensión afectiva.

4. Reforzando el trabajo en equipo, el debate, la negociación y la reflexión compartida.

\section{REFERENCIAS}

Aguilar, M. A. \& Castañón, N. (2014). Propuesta actividades y estrategias pedagógicas que promuevan una cultura de paz y capacidad de resolución de conflictos en instituciones educativas venezolanas, Revista de Comunicación de la SEECI, 34, 83-94. doi: https://doi.org/10.15198/seeci.2014.34.83-94

Catena, E (Ed.) (1989). Autobiografía. Gertrudis Gómez de Avellaneda. Poesías y epistolario de amor y de amistad. Madrid: Castalia.

Centro Reina Sofía sobre adolescencia y juventud (2015). Jóvenes y género. El estado de la cuestión. Madrid: Autor. Recuperado de http://adolescenciayjuventud.org/en/publications/monographs-andstudies/item/jovenes-y-genero.

De Celis, E. (2011). Prevención de la violencia de género. Prevenirla para erradicarla: actuando en las raíces de la violencia de género, en J. M. Pérez, y A. Escobar, (Coords.), Perspectivas de la violencia de género. Madrid: Grupo 5.

Díaz Aguado, M. J. (2002). Convivencia escolar y prevención de la violencia. MEC. Recuperado de http://www.deciencias.net/ convivir/1.documentacion/D.violencia/Diaz-aguado/index.html

Díaz Aguado, M.J. \& Carvajal Gómez, M.I. (2011). Igualdad y prevención de la violencia de género en la adolescencia. Madrid: Ministerio de Sanidad, Política Social e Igualdad.

Gairín Sallán, J.; Armengol Asparó, Carmen \& Silva García B. P. (2013). El «bullying» escolar. Consideraciones organizativas y estrategias para la intervención, Educación XX1, 16(1), pp. 17-38. doi: 10.5944/educxx1.16.1.715. 
Gómez Jarabo, I. y Sánchez Delgado, P. Formación del profesorado en cuestiones de género.

Gómez-Jarabo, I. (2015). Formación del profesorado para el tratamiento educativo de los conflictos sobre diversidad cultural y de género (tesis doctoral). Madrid: Universidad Complutense de Madrid.

Instituto de la Mujer/ Red2 Consultores (2004). Estudio para conocer la situación actual de la educación para la igualdad en España. Madrid: Instituto de la Mujer. Recuperado de http://www.educarenigualdad.org/upload/Doc_15_red2red.pdf

Otero, L. (2001). He aquí la esclava del señor: de cómo la mujer fue educada para el sacrificio y la sumisión. Barcelona: Ediciones B.

Pérez, J.M. \& Montalvo, A. (Coords.) (2011). Violencia de género. Prevención, detección y atención. Madrid: Grupo 5.

Reichardt, CH.S \& Cook, T.D. (1986). Hacia una superación del enfrentamiento entre los métodos cualitativos y cuantitativos, en T. D. Cook, y CH. S. Reichardt, Métodos cualitativos y cuantitativos en investigación evaluativa. Madrid: Morata.

Romero, A. \& Abril, P. (2008). Género y la formación del profesorado en los estudios de Educación Infantil, Revista Electrónica Interuniversitaria de Formación del Profesorado, 11(3), 40-50. Recuperado de http://www.aufop.com/aufop/home/

Sánchez, P.; Gonzalo, V.; Barrigüete. L.M. \& López, J. (2005). La violencia en la Educación Secundaria Obligatoria. Análisis de la situación y propuesta de intervención educativa. Granada: GEU.

Seminario Galego Educación Paz (2006). Educación emocional y violencia contra la mujer Estrategias para el aula. Madrid: La Catarata.

Subirats, M. (1994). Conquistar la igualdad: la coeducación hoy, Revista Iberoamericana de Educación, 6, 49-78.

\section{AUTORES}

\section{Inmaculada Gómez Jarabo}

Licenciada y Doctora en Pedagogía por la Universidad Complutense de Madrid, con experiencia como docente tanto en educación formal como no formal. Actualmente trabaja como profesora asociada del Departamento de Didáctica y Organización Escolar de la UCM y como profesional autónoma (dedicada al perfeccionamiento profesional). Líneas prioritarias de trabajo e investigación: Interculturalidad, género, convivencia, formación inicial y permanente del profesorado y TIC aplicadas a la educación.

http://orcid.org/0000-0003-0159-6910 
Gómez Jarabo, I. y Sánchez Delgado, P. Formación del profesorado en cuestiones de género

\section{Primitivo Sánchez Delgado}

Profesor Titular de Universidad en el Departamento de DOE de la Universidad Complutense (UCM). Licenciado y Doctor en CC. de la Educación con Premio Extraordinario por la UCM. Licenciado en $\mathrm{F}^{\mathrm{a}}$ y Letras $\left(\mathrm{H}^{\mathrm{a}}\right)$ por la Universidad de Salamanca. Ha sido profesor en todos los niveles del sistema educativo. Líneas de investigación: Desarrollo personal y profesional de docentes y educadores, interculturalidad, inclusión, educación para la paz.

http://orcid.org/0000-0001-7611-407X 